\title{
Effects of Nutritional Factors on the Formation of Ubiquinone by Tobacco Plant Cells in Suspension Culture ${ }^{\dagger}$
}

\author{
Tsutomu IKeda, Takashi Matsumoto and Masao NoguchI \\ Central Research Institute, The Japan Tobacco and Salt Public Corporation, \\ 6-2, Umegaoka, Midori-ku, Yokohama 227, Japan
}

Received March 19, 1976

\begin{abstract}
The ubiquinone (UQ) content of BY-2 cells on surface culture was examined to compare with that in suspension culture. The UQ content on surface culture was a little lower than that in suspension culture, but the pattern of the time-course of the UQ formation on surface culture was similar.

The changes of UQ content in BY-2 cells during autolysis were also examined. UQ in the cells subjected to autolysis was not rapidly metabolized nor excreted into the medium.

In order to obtain basic information for UQ formation by BY-2 cells in suspension culture, the cultural conditions, especially nutritional ones were investigated. Addition of 2,4-D was remarkably effective on UQ formation and a higher UQ content was observed with a higher 2,4-D concentration. Sucrose and glucose concentrations in the original medium were also influential factors. The UQ content tends to increase with the decrease of the sugar content. Precursors of UQ, amino acids, vitamins and organic acids were not effective on the UQ formation.
\end{abstract}

Since Bonner tried to produce gum using guayule (Parthenium argentatum) callus, ${ }^{1)}$ many plants have been investigated to make them produce secondary metabolites by tissue culture. Many plant calli have been induced and investigated on their chemical constituents and cultural conditions. Steward ${ }^{2}$ and Hildebrandt ${ }^{3}$ proved the totipotency of cultured cells by raising the normal plant from a cultured single cell. It is suggested that cultured cells also have the latent ability of their metabolites formation as their parent plants. However, cultured cells usually do not produce useful secondary products of their parent plants at all. Even if those are produced, the contents are very low as compared with those of the parent plants, although a considerable production of shikonins, ${ }^{4)}$ anthocyanin $^{5)} 1$-maackianin ${ }^{6 /}$ and monoterpenes ${ }^{7)}$ have been reported.

In our previous paper, ${ }^{8,9)}$ the isolation of

$\uparrow$ Studies on the Culture Conditions of Higher Plant Cells in Suspension Culture. Part VIII, see references $5,9)$.

Abbreviations used in this paper: UQ., Ubiquinone; 2,4-D., 2,4-dichlorophenoxyacetic acid.
UQ-10 from the cultured cells of Nicotiana tabacum L. c.v. BY-2 and the time-course change in the UQ content of BY-2 cells during growth were reported. According to our investigations, BY-2 cells seem to contain UQ-10 much more than their parent plants and the UQ-10 productivity of the cultured BY-2 cells are similar to that of the microorganisms which have been reported to contain much UQ-10. Therefore, BY-2 cells might be a suitable source for a large scale production of UQ-10. It is interesting that UQ which is an essential component of living cells is accumulated relatively much in cultured cells. Few attempts have been made for the production of any useful primary metabolite ${ }^{10}$ by plant cell culture, probably because these productivities of cultured cells seem to be considerably inferior to those of microorganisms or parent plants.

Many investigations on the cultural conditions for UQ formation have been carried out using microorganisms, ${ }^{11 \sim 14)}$ especially hydrocarbon assimilating yeast (Candida tropicalis) by Shimizu et al., ${ }^{15 \sim 19)}$ but few such studies 
with cultured plant cells have been reported. In order to obtain basic information for the UQ production by cultured cells, cultural conditions, especialy nutritional ones were investigated and this paper deals with the results.

\section{MATERIALS AND METHODS}

1) Cultured cells. Cultured tobacco cells were induced from the pith of Nicotiana tabacum L. c.v. BY-2 by Kawashima in 1968 at Hatano Tobacco Experiment Station. Stock suspension culture was routinely subcultured on Linsmaier-Skoog medium ${ }^{20)}$ at a transfer interval of 7 days.

2) Culture medium. Linsmaier-Skoog inorganic medium $^{20)}$ added with (per liter) $30 \mathrm{~g}$ of sucrose, $1.0 \mathrm{mg}$ of thiamine- $\mathrm{HCl}, 100 \mathrm{mg}$ of myo-inositol and $0.2 \mathrm{mg}$ of 2,4-D was used as the basal medium. The initial $\mathrm{pH}$ of the medium was adjusted to 6.0 with $0.5 \mathrm{~N}$ sodium hydroxide.

3) Surface culture. Surface culture of BY-2 cells was carried out in the petri dish $(\phi 15 \times 3.5 \mathrm{~cm})$ containing $100 \mathrm{ml}$ of the basal medium $(0.8 \%$ agar $)$ for 30 days at $28^{\circ} \mathrm{C}$ in the dark. Seed cultures (suspension culture) were incubated for 7 days and $10 \mathrm{ml}$ of the culture was inoculated into each petri dish.

4) Culture conditions and harvesting method of $B Y_{-}$ 2 cells. The culture conditions and harvesting method of $\mathrm{BY}-2$ cells were the same as those described in the previous paper. ${ }^{8}$ In the case of examining changes in UQ content during autolysis, the cells which had been cultured for 8 days were left in flasks without shaking at $28^{\circ} \mathrm{C}$ in the dark.

5) Extraction and determination of $U Q$. Extraction and determination of $\mathrm{UQ}$ were carried out as described in the previous paper. ${ }^{97}$

\section{RESULTS AND DISCUSSION}

1) UQ formation in surface-cultured cells on the agar medium

The time-course of UQ formation with cell growth of BY-2 on surface culture is shown in Fig. 1. The cell mass in dry weight reached the maximum yield at 13 days after inoculation and showed 1.50 g-dry weight per petridish and the fresh weight at 18 days showed $58.3 \mathrm{~g}$. The UQ content of BY-2 cells increased toward the stationary phase, reaching

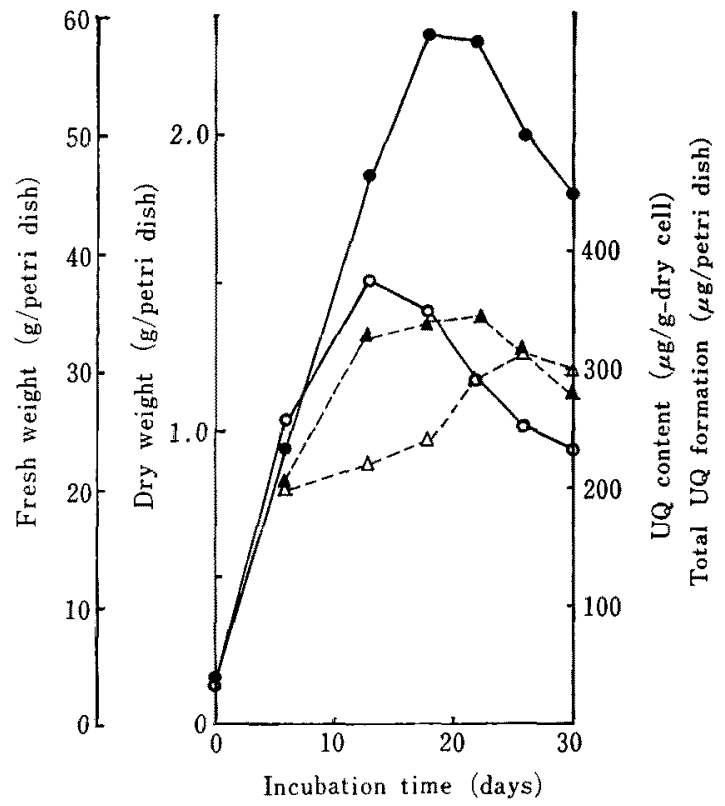

FIG. 1. Cell Growth and UQ Formation in BY-2 Cells on Surface Culture.

As for culture medium and cultural conditions, see the text.

Cell growth: $-\longrightarrow$, fresh weight; $0-0$, dry weight $\triangle \cdots \triangle$, UQ content; $\triangle \cdots-\cdots$, total UQ.

the highest level (313 $\mu \mathrm{g}$ per g-dry weight) on the 26th day.

The growth rate on the surface culture was about a half that of suspension culture in both dry weight and fresh weight. The UQ content on surface culture was a little lower than that of suspension culture. The pattern of the timecourse of the UQ formation on the surface culture was similar to that of suspension culture.9) Thomas and Stobart reported a similar result for Kalanchoë crenata callus. ${ }^{21}$ It seems that there is no difference between suspension culture and surface culture in the pattern of UQ formation.

\section{2) Changes in the $U Q$ content of $B Y-2$ cells} during autolysis

Changes of some chemical components during the autolysis of $\mathrm{BY}-2$ cells have been reported by Koiwai et al. ${ }^{22}$ In order to see whether the UQ in harvested cells is metabolically stable or not, changes in its content during the autolysis of the cells were examined. Results are shown in Fig. 2. The initial cell 


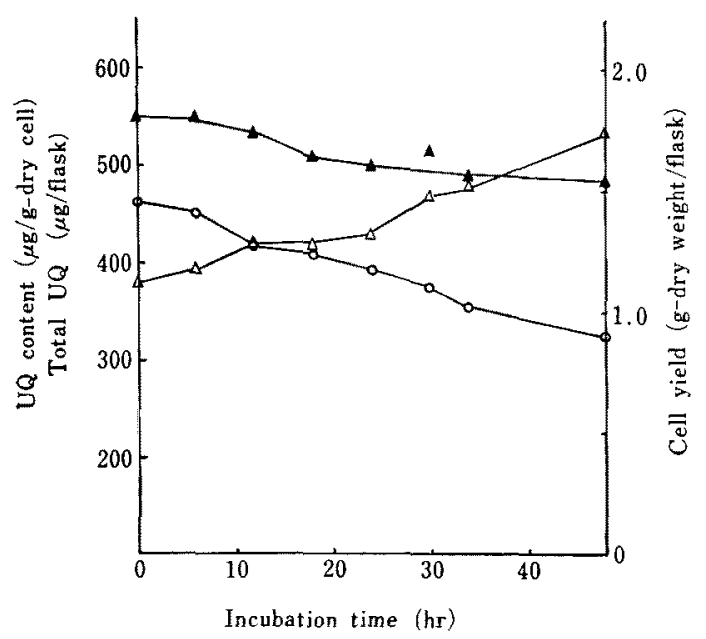

FIG. 2. Changes of UQ Content in BY-2 Cells during Autolysis.

Cultured cells were used at 8 days after inoculation. As for culture medium and cultural conditions, see the text.

$\mathrm{O}-\mathrm{O}$, cell yield; $\triangle-\triangle$, UQ content; $\Delta-\Delta$, total UQ.

mass, 1.45 g-dry weight per flask, gradually decreased to $0.90 \mathrm{~g}$-dry weight per flask after $48 \mathrm{hr}$. On the contrary, the initial UQ content (380 $\mu \mathrm{g}$ per g-dry weight) gradually increased to $540 \mu \mathrm{g}$ per g-dry weight after $48 \mathrm{hr}$. Total UQ per flask was $551 \mu \mathrm{g}$ at first, $500 \mu \mathrm{g}$ after $24 \mathrm{hr}$ and $486 \mu \mathrm{g}$ after $48 \mathrm{hr}$ respectively. This small decrease may be due to either excretion of UQ into the medium or its digestion in cells. These results suggest that UQ in the cells during autolysis was not rapidly metabolized nor excreted. Therefore, the UQ content seems not to change during sampling and storage.

\section{3) Effect of 2,4-D}

In order to investigate the effect of the concentration of 2,4-D on UQ formation, the UQ content and cell yield were examined in the media containing various concentrations of 2,4-D. Results are shown in Fig. 3. The UQ content in BY-2 cells tends to increase at higher concentrations of 2,4-D, while the cell yield decreased a little at higher 2,4-D concentrations. The maximum yield of UQ per flask was $664 \mu \mathrm{g}$ in the medium with 2.0 $\mathrm{mg}$ per liter of 2,4-D.

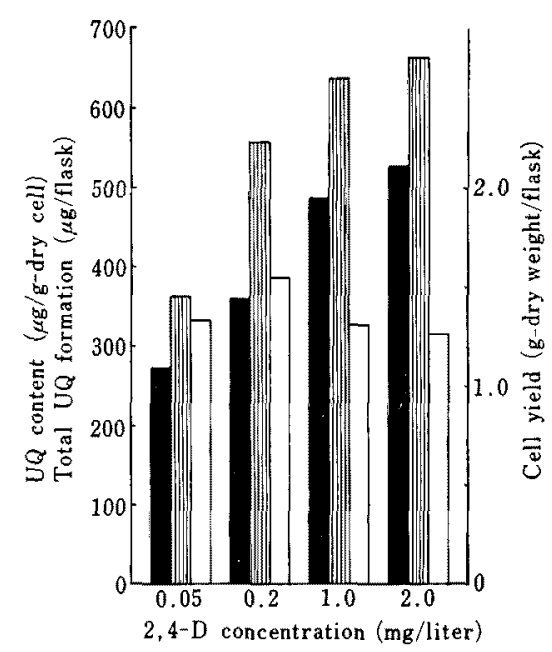

Fig. 3. Effect of 2,4-D Concentration on the Formation of UQ.

Cultured cells were harvested at 8 days after inoculation. As for culture medium and cultural conditions except for 2,4-D concentrations, see the text.

घ, UQ content; 佂, total UQ; $\square$, cell yield.

In the previous paper, ${ }^{9}{ }^{\prime}$ we reported that the UQ content of crown gall cells which are auxin non-requiring for their rapid growth was much lower than those of BY-2 and Xanthi cells. Therefore, 2,4-D seems to promote the biosynthesis of UQ either directly or indirectly. On the other hand, 2,4-D has been reported to repress the formation of such secondary products as nicotine, ${ }^{23)}$ anthocyanin $^{5}$ and shikonins ${ }^{4}$ in comparison to $\beta$-indoleacetic acid and $\alpha$-naphthaleneacetic acid at low concentrations. It seems very interesting that $U Q$, which is an essential primary product was increased by high concentrations of 2,4-D.

\section{4) Effect of kinetin}

In order to investigate the effect of kinetin on UQ formation, the UQ content was examined in both media of low and high levels of kinetin, although kinetin is not required by $\mathrm{BY}-2$ cells for their growth. Results are shown in Table I. The UQ content was not affected by the low level of kinetin but about a $30 \%$ decrease was observed by the high level of kinetin. Kinetin seems to have some inhibitory effect on UQ formation. 
TABLE I. EFFECT OF KINETIN ON UQ Formation BY BY-2 CeLls

Cultured cells were harvested at 8 days after inoculation. As for culture medium and cultural conditions, see the text.

\begin{tabular}{cccc}
\hline \multirow{2}{*}{$\begin{array}{c}\text { Kinetin } \\
\text { concentration } \\
(\mathrm{mg} / \text { liter })\end{array}$} & $\begin{array}{c}\text { Cell yield } \\
\text { (g-dry } \\
\text { wt./flask) }\end{array}$ & \begin{tabular}{c} 
UQ formation \\
\cline { 3 - 4 } cell)
\end{tabular} & $(\mu \mathrm{g} /$ flask) \\
\hline 0 & 1.52 & 393 & 597 \\
0.2 & 1.39 & 387 & 538 \\
2.0 & 1.65 & 280 & 462 \\
\hline
\end{tabular}

\section{5) Effect of sugars}

The effect of sugar concentration was examined on UQ formation by varying the initial concentration stepwise from 2 to $5 \%$. As shown in Fig. 4, the UQ content was the highest with $2 \%$ sugar concentration and the lowest with $5 \%$ concentration both in sucrose and glucose media. No considerable difference was observed between sucrose and glucose as carbon sources in UQ formation. The UQ content showed a tendency to increase with lower sugar concentrations.

In microorganisms, a similar result was recognized with Saccharomyces cerevisiae by Gordon and Stewart. ${ }^{14}$ Our results suggest that UQ formation in BY-2 cells is also subject to catabolite repression as observed in microorganisms under the aerobic condition.
They recognized an inverse relationship between the UQ content of the cells and the glucose concentration in the yeast which was grown in batch culture with $5.4 \%$ glucose as the carbon source. In addition, the highest content of UQ was observed after the glucose was exhausted. In our experiments, the UQ content increased, reaching the highest level at the stationary phase where the residual sucrose was very little or exhausted. This phenomenon seems to be due to the decrease in sugar concentration as in the case of microorganisms. In the case of hydrocarbon assimilating microorganisms, UQ was produced in the hydrocarbon medium much more than in the glucose medium. ${ }^{15}$ It seems that in the hydrocarbon medium, UQ formation may be free from catabolite repression which maintains a low UQ level. This control is presumably exerted at the level of the activity of the biosynthetic pathway for UQ. From these results, it seems that carbon sources play an important role on UQ formation.

As seen from Fig. 4, the yield of UQ per sugar consumed increased with the decrease in the initial sugar concentration.

\section{6) Effect of sucrose and inorganic nutrients concentrations}

Figure 5 shows the influence of Linsmaier-

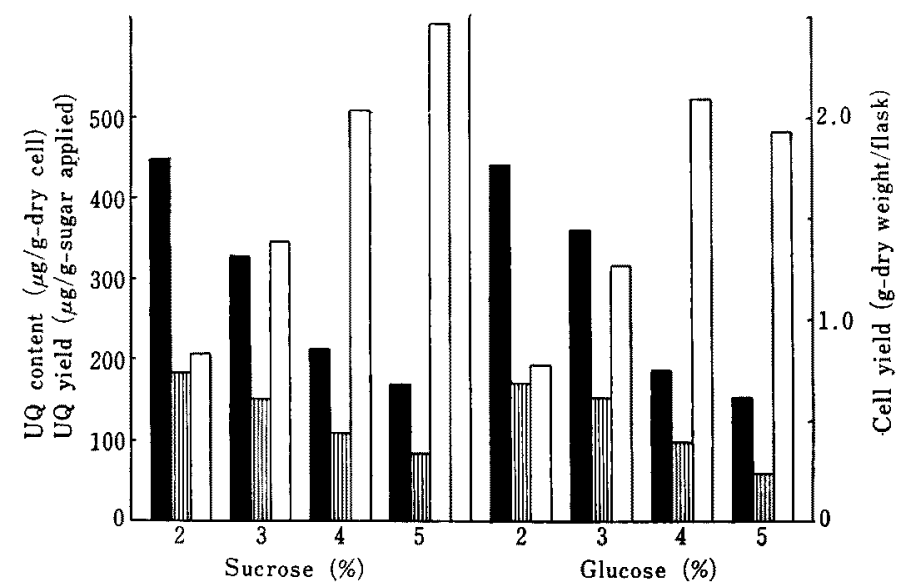

FIG. 4. Effect of Sucrose and Glucose Concentrations on the UQ Formation.

Cultured cells were harvested at 8 days after inoculation. As for culture medium and cultural conditions except for sugar concentrations, see the text.

n, UQ content; $\mathbb{M}$, UQ yield; $\square$, cell yield. 


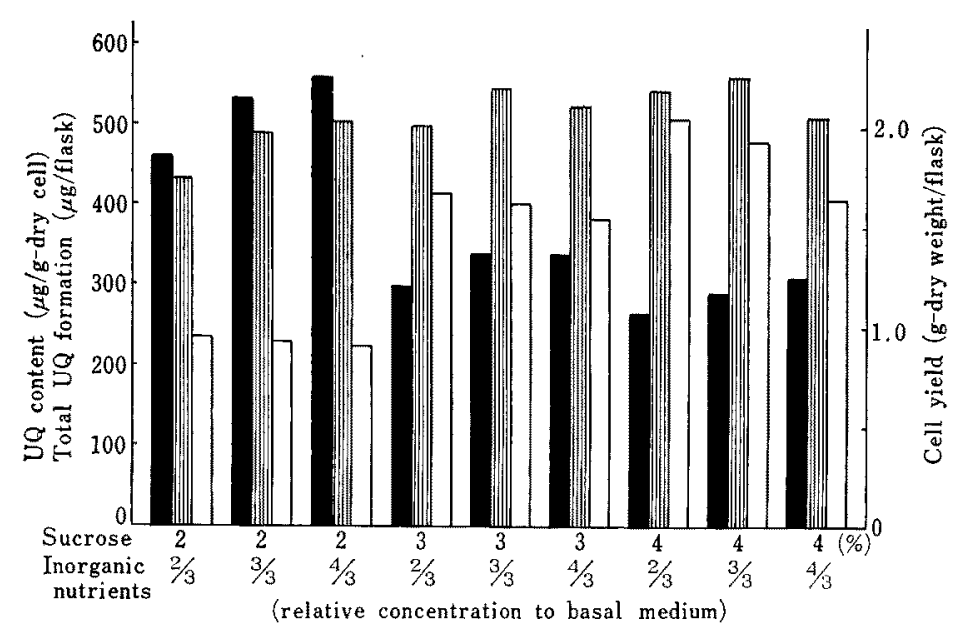

FIG. 5. Effect of Sucrose and Inorganic Nutrients Concentrations on the UQ Formation.

Cultured cells were harvested at 8 days after inoculation. As for culture medium and cultural conditions except for sucrose and inorganic nutrients concentrations, see the text.

m, UQ content; 四, total UQ; $\square$, cell yield.

Skoog inorganic nutrients concentrations on UQ formation in the presence of various sucrose concentrations. The cell yield tends to decrease at higher concentrations of inorganic nutrients, especially in the presence of higher concentrations of sucrose. The UQ content of cells tends to increase with the increase in inorganic nutrients. However, this increase of the UQ content was less significant than that caused by the decrease of sugar concentration and may be caused by a relative decrease of sugar concentration. Accordingly, UQ formation in BY-2 cells seems to be remarkably affected by sugar concentration in the medium rather than inorganic nutrients.

\section{7) Effect of precursors}

The biosynthetic pathway of UQ has been studied by many workers using various microorganisms ${ }^{24 \sim 26)}$ and plant tissues. ${ }^{27 \sim 29}$ ? It has been well confirmed that the aromatic moiety of UQ is derived from phosphoenolpyruvate through shikimic and $p$-hydroxybenzoic acid. The effect of the precursors of the aromatic moiety in UQ molecule was investigated by respective addition of phenylalanine, tyrosine, trans-cinnamic acid, $p$-coumaric acid, $p$-hydroxybenzaldehyde and $p$ hydroxybenzoic acid to the medium at three different levels $(5,10,50 \mathrm{mg}$ per liter). The content of BY-2 cells showed no change by the addition of any one of these precursors. Therefore, in BY-2 cells, a deficiency of any aromatic precursors is not a limiting factor of UQ synthesis unless they are not taken up into the cells. In Candida tropicalis, ${ }^{16)} p$ hydroxybenzoic acid, the direct precursor of UQ, and tyrosine were very effective on UQ formation in the hydrocarbon medium, while phenylalanine was not. However, when glucose was used as the carbon source, no remarkable effect of these precursors was observed. Considering these results, the positive effect of these precursors on UQ formation does not seem to come out when the carbon source is in such a high oxidized state as sucrose.

\section{8) Effect of other organic substances}

Amino acids were added at $0.5 \mathrm{~mm}$ concentration to the basal medium to examine their effects on UQ formation. Pactor and Glover reported $^{11)}$ that when the culture medium of Aspergillus fumigatus was supplemented with such branched-chain amino acids as L-leucine, L-isoleucine and L-valine, UQ production was increased. Furthermore, only L-phenylalanine among the aromatic amino acids stimulated 
UQ formation. L-Methionine, which is supposed to play a role in the methylation of the aromatic ring, was also effective on UQ formation. However, in our experiments, the UQ content of BY-2 cells did not increase by the addition of any amino acids.

Vitamins (0.1 $\mathrm{mg}$ and $0.5 \mathrm{mg}$ per liter) i.e. $p$-aminobenzoic acid, biotin, cyanocobalamin, folic acid, DL- $\alpha$-lipic acid, nicotinic acid, Capantothenate, pyridoxine- $\mathrm{HCl}$ and riboflavin, which were not required for the growth of the cells, were not effective on UQ formation in $\mathrm{BY}-2$ cells.

Thiamine- $\mathrm{HCl}$ which is required for the cell growth also gave no effect on UQ formation within the concentration range from $1 \mathrm{mg}$ to $100 \mathrm{mg}$ per liter of the medium.

Organic acids i.e. fumaric, L-malic, citratic, succinic, acetic and malonic acids were independently added at $1 \mathrm{~mm}$ and $10 \mathrm{~mm}$ concentrations to the basal medium and DL-lactic acid was added at $2 \mathrm{~mm}$ and $20 \mathrm{~mm}$. The UQ content of BY-2 cells was not increased by the addition of these organic acids.

Acknowledgement. The authors wish to express their thanks to Mrs. Y. Hashimoto in our laboratory for supplying seed cultured cells.

\section{REFERENCES}

1) B. Arreguin and J. Bonner, Arch. Biochem., 26, 178 (1950).

2) F. C. Steward, Am. J. Bot., 45, 709 (1958).

3) V. Vasil and A. C. Hildebrandt, Science, 150, 889 (1965).

4) M. Tabata, H. Mizukami, N. Hiraoka and M. Konoshima, Phytochemistry, 13, 927 (1974).

5) T. Matsumoto. K. Nishida, M. Noguchi and E. Tamaki, Agr. Biol. Chem., 37, 561 (1973).

6) T. Furuya and A. Ikuta, Chem. Pharm. Bull., 16, 771 (1968).
7) H. Sugisawa and Y. Ohnishi, Agr. Biol. Chem., 40, 231 (1976).

8) T. Ikeda, T. Matsumoto, K. Kato and M. Noguchi, Agr. Biol. Chem., 38, 2297 (1974).

9) T. Ikeda, T. Matsumoto and M. Noguchi, Phytochemistry, 15, 568 (1976).

10) A. Koiwai, M. Noguchi and E. Tamaki, ibid. 10, 561 (1971).

11) N. M. Packter and J. Glover, Biochim. Biophys. Acta, 58, 531 (1962).

12) M. D. Henninger, Biochem. Biophys. Res. Commun., 19, 233 (1965).

13) G. R. Whistance and D. R. Threlfall, Biochem. J., 108, 505 (1968).

14) P. A. Gordon and P. R. Stewart, Biochim. Biophys. Acta, 177, 358 (1969).

15) S. Shimizu, A. Tanaka and S. Fukui, J. Ferment. Technol., 47, 542 (1969).

16) S. Shimizu, A. Tanaka and S. Fukui, ibid., 47, 551 (1969).

17) S. Shimizu, A. Tanaka and S. Fukui, ibid., 48, 533 (1970).

18) S. Shimizu, A. Tanaka and S. Fukui, ibid., 48, 542 (1970).

19) S. Shimizu, A. Tanaka and S. Fukui, ibid., 48, 549 (1970).

20) E. M. Linsmaier and F. Skoog, Physiol. Plantarum., 18, 100 (1965).

21) D. R. Thomas and A. K. Stobart, New Phytol, 70, 163 (1971).

22) A. Koiwai, Y. Shiozawa and M. Noguchi, Agr. Biol. Chem., 36, 971 (1972).

23) T. Furuya, H. Kojima and K. Syono, Phytochemistry, 10, 1529 (1971).

24) G. R. Whistance, J. F. Dillon and D. R. Threlfall, Biochem. J., 111, 461 (1969).

25) G. R. Whistance, B. S. Brown and D. R. Threlfall, ibid., 117, 119 (1970).

26) Ah Law, D. R. Threlfall and G. R. Whistance, ibid., 123, 331 (1971).

27) G. R. Whistance, D. R. Threlfall and T.W. Goodwin, ibid., 105, 145 (1967).

28) W. T. Griffiths, D. R. Threlfall and T.W. Goodwin, European J. Biochem., 5, 124 (1968).

29) D. R. Threlfall and G. R. Whistance, Phytochemistry, 9, 355 (1970). 